NASA Technical Memorandum 105663

ICOMP-92-10; CMOTT-92-06.

\title{
Kolmogorov Behavior of Near-Wall Turbulence and Its Application in Turbulence Modeling
}

Tsan-Hsing Shih

Institute for Computational Mechanics in Propulsion and Center for Modeling of Turbulence and Transition Lewis Research Center.

Cleveland, Ohio

and

John L. Lumley

Cornell University

Ithaca, New York
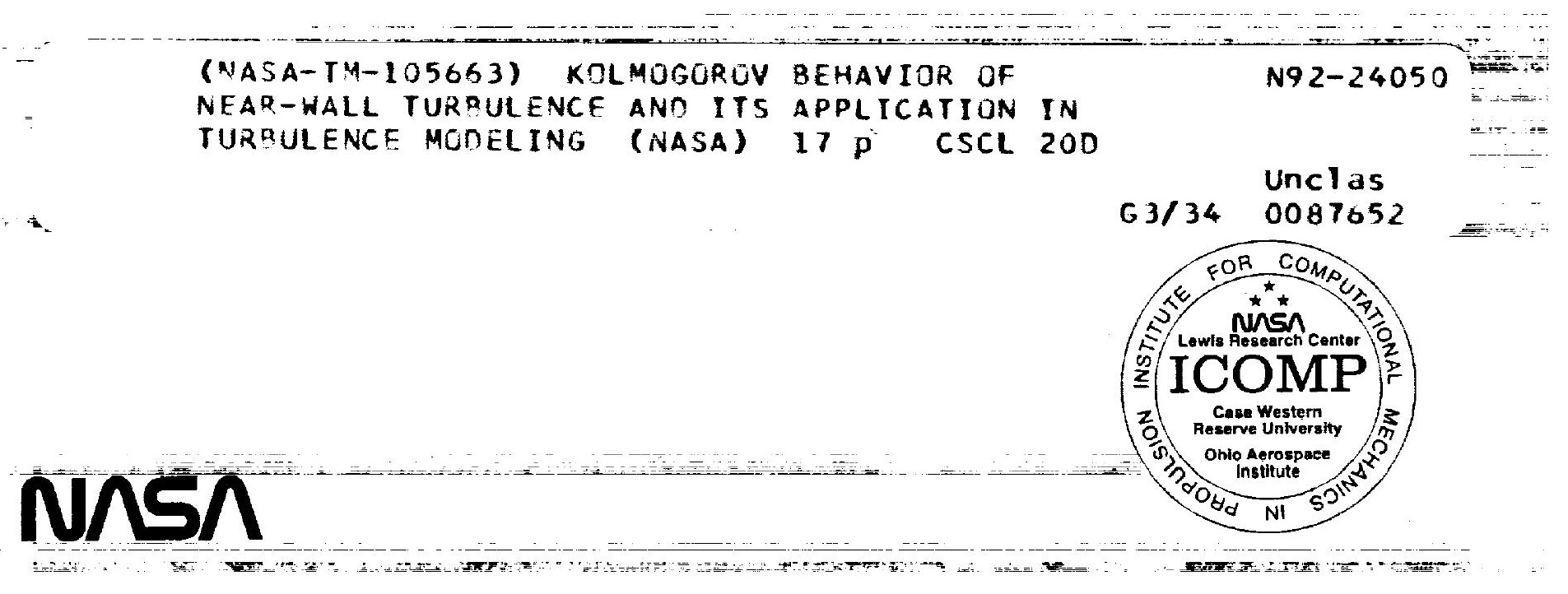


\title{
Kolmogorov Behavior of Near-Wall Turbulence and Its Application in Turbulence Modeling
}

\author{
Tsan-Hsing Shih* \\ Institute for Computational Mechanics in Propulsion \\ and Center for Modeling of Turbulence and Transition \\ Lewis Research Center \\ Cleveland, Ohio 44135 \\ John L. Lumley \\ Cornell University \\ Ithaca, New York 14853
}

\begin{abstract}
The near-wall behavior of turbulence is re-examined in a way different from that pro-

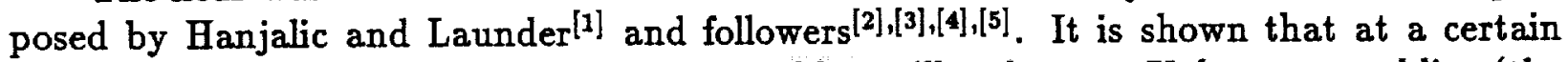
distance from the wall, all energetic large eddies will reduce to Kolmogorov eddies (the smallest eddies in turbulence). All the important wall parameters, such as friction velocity, viscous length scale, and mean strain rate at the wall, are characterized by Kolmogorov microscales. According to this Kolmogorov behavior of near-wall turbulence, the turbulence quantities, such as turbulent kinetic energy, dissipation rate, etc. at the location where the large eddies become "Kolmogorov" eddies, can be estimated by using both direct numerical simulation (DNS) data and asymptotic analysis of near-wall turbulence. This information will provide useful boundary conditions for the turbulent transport equations. As an example, the concept is incorporated in the standard $k-\varepsilon$ model which is then applied to channel and boundary layer flows. Using appropriate boundary conditions (based on Kolmogorov behavior of near-wall turbulence), there is no need for any wall-modification to the $k-\varepsilon$ equations (including model constants). Results compare very well with the DNS and experimental data.
\end{abstract}

\section{Kolmogorov behavior of near-wall turbulence}

It is well known that at a sufficiently high Reynolds number, $R_{e r}$, there are following relations for the near-wall turbulence in a channel flow:

$$
-\overline{u v}^{+}+\frac{d U^{+}}{d y^{+}}=1
$$

In the inertial sublayer $\left(y^{+}>30\right)$,

$$
\begin{aligned}
U^{+} & =\frac{1}{k} \log y^{+}+C \\
-\overline{u v}^{+} & \approx 1
\end{aligned}
$$

In the viscous sublayer $\left(y^{+}<5\right)$,

$$
\begin{aligned}
U^{+} & =y^{+} \\
-\overline{u v}^{+} & \approx 0
\end{aligned}
$$

*Work funded under NASA Cooperative Agreement 3-233. 
where

$$
\begin{aligned}
U^{+} \equiv \frac{U}{u_{\tau}}, & \overline{u v}{ }^{+} \equiv \frac{\overline{u v}}{u_{\tau}^{2}} \\
y^{+} \equiv \frac{u_{\tau} y}{\nu} & R_{e \tau} \equiv \frac{u_{\tau} h}{\nu}
\end{aligned}
$$

and $k=0.4$ is von Karman's constant, $u_{\tau}$ is the friction velocity, $h$ is the channel half width, $y$ is the distance from the wall, $U$ and $\overline{u v}$ are the mean velocity and turbulent shear stress respectively. The Kolmogorov length, velocity and time microscales of turbulence are

$$
\eta=\left(\frac{\nu^{3}}{\varepsilon}\right)^{\frac{2}{4}}, \quad v=(\nu \varepsilon)^{\frac{1}{4}}, \quad \tau=\left(\frac{\nu}{\varepsilon}\right)^{\frac{1}{2}}
$$

where $\nu$ is the kinematic viscosity, and $\varepsilon$ is the dissipation rate of turbulent kinetic energy. Equations (1)-(6) will be used intensively in analyzing the near-wall behavior of turbulence.

Now let us show that the important wall parameters, such as the friction velocity $u_{\tau}$, the viscous length scale $\nu / u_{\tau}$, and the mean strain rate at the wall, are actually characterized by Kolmogorov microscales. We may use Eq.(6) to write,

$$
\frac{u_{\tau} \eta}{\nu}=\frac{u_{\tau}}{\nu}\left(\frac{\nu^{3}}{\varepsilon}\right)^{\frac{1}{4}}=\left(\frac{1}{\varepsilon^{+}}\right)^{\frac{1}{4}}
$$

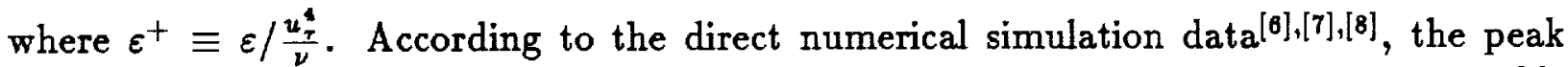
value of $\varepsilon^{+}$near the wall increases slowly from 0.165 to 0.251 with increasing Reynolds number (based on the momentum thickness), and tends to an asymptotic value (see Fig.1). These DNS data show that $\frac{u_{\tau} \eta}{\nu}$ is of order one. If we take 0.251 as the asymptotic value of $\varepsilon^{+}$at high Reynolds numbers, the value of $\frac{u_{\tau} \eta}{\nu}$ is about 1.413. This means that very near the wall

$$
u_{\tau} \approx 1.413 v
$$

because $v \eta / \nu$ is always one. Equation (7) can be also shown in the following way:

$$
v=(\nu \varepsilon)^{\frac{1}{4}} \approx\left(\nu \frac{u_{\tau}^{3}}{k y}\right)^{\frac{1}{4}}=\left(\frac{1}{k y^{+}}\right)^{\frac{1}{4}} u_{\tau}
$$

where we have estimated $\varepsilon$ with $u_{\tau}^{3} / k y$, which is accurate in the inertial sublayer, and may still give the order of magnitude of $\varepsilon$ further toward (but not at) the wall. Eq.(8) shows that $v$ is indeed of order $u_{\tau}$ near the wall where $k y^{+}$is of order 1 .

The viscous length is defined as $\nu / u_{\tau}$. If $u_{\tau}$ is estimated with $v$, we obtain

$$
\frac{\nu}{u_{\tau}} \approx \frac{\nu}{v}=\frac{\nu}{(\nu \varepsilon)^{\frac{1}{4}}}=\eta
$$

The mean strain rate at the wall is of order $\partial U / \partial y$. Using Eq.(4), we obtain

$$
\frac{\partial U}{\partial y}=\frac{u_{\tau}^{2}}{\nu} \approx \frac{v^{2}}{\nu}=\frac{(\nu \varepsilon)^{\frac{1}{2}}}{\nu}=\frac{1}{\tau}
$$


Equations (7)-(10) show that near the wall all important physical parameters of turbulence are characterized by Kolmogorov microscales. We shall see later that there is a narrow region where the distance from the wall is smaller than the Kolmogorov length scale. In that region, the turbulence can not be self-sustained because of the overwhelming viscous action, and the flow is dominated by the viscous stress. Therefore, it is not important that what is the behavior of turbulence within that region $(y<\eta)$. The important thing is the behavior of turbulence above that region. It is important to realize that approaching the wall, the turbulence is going to be described by Kolmogorov microscales, which are finite, and non-zero quantities. We will refer to this asymptotic property as Kolmogorov behavior of near-wall turbulence.

\section{Near-wall asymptotic turbulence quantities}

To study the near-wall behavior of turbulence, one needs first to find where (or at what distance from the wall) the size of energetic large eddies will reduce to Kolmogorov microscale, and second to estimate the turbulent quantities, such as the turbulent kinetic energy, the dissipation rate, etc., at that position.

Let $y_{\eta}$ indicate the position where the integral scale of turbulence $\ell$ equals the Kolmogorov length scale $\eta$. We examine the variations of these two scales as the wall is approached from the inertial sublayer. The size of energetic large eddies, or the integral scale of turbulence is of order $y$. In the inertial sublayer, it can be estimated as (see Tennekes \& Lumley $^{[9]}$ )

$$
\ell \approx k y
$$

For the Kolmogorov length scale $\eta$, if we estimate $\varepsilon$ with $u_{\tau}^{3} / k y$ (which is quite accurate in the inertial sublayer), we obtain

$$
\eta \approx\left(\frac{\nu^{3} k y}{u_{\tau}^{3}}\right)^{\frac{1}{4}}
$$

Equations (11) and (12) show that both $\ell$ and $\eta$ decrease toward the wall, but at different rates. The integral scale $\ell$ decreases much faster than $\eta$ does. We may imagine that at some point near the wall, $\ell$ will approach $\eta$. We refer to this point as the limit point of turbulence, because passing beyond this point toward the wall, the turbulence is no longer important. Now if we use Eq.s (11) and (12), and let $\ell$ equal $\eta$, we obtain

$$
\left(k \frac{u_{\tau} y}{\nu}\right)^{\frac{3}{4}}=1
$$

Equation (13) indicates that the limit point of turbulence is

$$
y_{\eta}^{+} \approx \frac{1}{k}=2.5
$$

The numerical value of $y_{\eta}^{+}$here should not be taken too seriously. It is an order of magnitude value, because we have assumed $\varepsilon=u_{\tau}^{3} / k y$ which is accurate only in the inertial 
sublayer, and also this value can be changed by the point we pick for the viscous cutoff in the spectrum. It is reasonable to choose the value at the top of the viscous sublayer:

$$
y_{\eta}^{+} \approx 6
$$

In the region where $y<y_{\eta}$, the self-sustaining turbulence cannot exist. Here we define turbulence to be a self-sustaining chaotic motion of the fluid, dominated by inertia, so that the scale of the dissipation is at least somewhat smaller than the scale of the energy containing eddies. As viscous effects become stronger, and the two scales approach each other, a point comes at which the chaotic motion can no longer sustain itself, but must depend on energy imported from neighboring regions. The chaotic disturbances of the viscous sublayer are of this nature; by our definition they are not turbulent, although surely chaotic, since they depend on the truly turbulent motions in the buffer layer and beyond for their energy.

The physical importance of Eq.(14) is that there exists a turbulence limit point $y_{\eta}$ which is not zero. To study the asymptotic behavior of turbulence near the wall is to find the behavior of turbulence at this turbulence limit point.

Now let us estimate the turbulence quantities at the point $y_{\eta}$. First, let us look at the dissipation rate at that point, $\varepsilon_{\eta}$. In the inertial sublayer, $\varepsilon$ is well represented by $u_{\tau}^{3} / k y$ or $\left(u_{\tau}^{4} / \nu\right)\left(1 / k y^{+}\right)$which shows that the dissipation rate increases toward the wall. If we extrapolate this relation to the limit point $y_{\eta}$, we obtain that $\varepsilon_{\eta}$ is of order $u_{\tau}^{4} / \nu$. On the other hand, if we use Eq.(7) at $y_{\eta}$,

$$
v_{\eta}=\frac{u_{\tau}}{1.413}=\left(\nu \varepsilon_{\eta}\right)^{\frac{1}{4}}
$$

we obtain

$$
\varepsilon_{\eta}=0.251 \frac{u_{\tau}^{4}}{\nu}
$$

This means that according to the DNS data, $\varepsilon_{\eta}$ is indeed of order $u_{\tau}^{4} / \nu$. Eq.(16) gives the peak value of the dissipation rate near the wall.

At the turbulence limit point, the characteristic velocity is $v_{\eta}$, therefore the turbulent kinetic energy $K$ at $y_{\eta}$ can naturally be estimated as

$$
K_{\eta}=\frac{1}{2} v_{\eta}^{2}=\frac{1}{2}\left(\frac{u_{\tau}}{1.413}\right)^{2}=0.250 u_{\tau}^{2}
$$

For the turbulent shear stress $-\overline{u v}$, if Eq.(1) and (4) are used at $y_{\eta}$, we obtain

$$
-(\overline{u v})_{\eta}=0
$$


Other shear stresses should also vanish at $y_{\eta}$. Finally, the energy components $\overline{u^{2}}, \overline{v^{2}}$, and $\overline{w^{2}}$ are not equal because of the anisotropy of turbulence near the wall. If we use the experimental data ${ }^{[9]}$ in the inertial sublayer to estimate the anisotropy at $y_{\eta}$, i.e.

$$
\begin{aligned}
& \frac{\overline{u^{2}}}{\overline{\overline{v^{2}}}}=a \approx 6.250 \\
& \frac{\overline{w^{2}}}{\overline{v^{2}}}=b \approx 3.625
\end{aligned}
$$

and $\overline{u^{2}}+\overline{v^{2}}+\overline{w^{2}}=2 K$, we obtain

$$
\begin{aligned}
& \overline{u^{2}}=\frac{a}{a+b+1} 2 K \\
& \overline{v^{2}}=\frac{1}{a+b+1} 2 K \\
& \overline{w^{2}}=\frac{b}{a+b+1} 2 K
\end{aligned}
$$

Using Eq.(17), we may estimate these energy components at $y_{\eta}$ :

$$
\begin{aligned}
& \left(\overline{u^{2}}\right)_{\eta} \approx 0.287 u_{\tau}^{2} \\
& \left(\overline{v^{2}}\right)_{\eta} \approx 0.046 u_{\tau}^{2} \\
& \left(\overline{w^{2}}\right)_{\eta} \approx 0.167 u_{\tau}^{2}
\end{aligned}
$$

Equations (14), (16), (17), (18), and (21) represent the near-wall asymptotic behavior of turbulence. These equations provide useful information about the boundary conditions for turbulent transport equations such as $K-\varepsilon$ equations, Reynolds stress equations, etc. These ideas can be used at any level of turbulence modeling. In the next two sections, we illustrate how they can be very easily incorporated into the eddy viscosity $K-\varepsilon$ models.

\section{Eddy viscosity}

Eddy viscosity (for incompressible flows) is defined as

$$
-\overline{u_{i} u_{j}}=\nu_{T}\left(U_{i, j}+U_{j, i}\right)-\frac{1}{3} \delta_{i j} \overline{u_{k} u_{k}}
$$

For a two-dimensional channel flow, it becomes

$$
-\overline{u v}=\nu_{T} \frac{d U}{d y}
$$

Note that Equation (1) is valid for any finite $y^{+}$(but not for $y^{+} \rightarrow \infty$ at $R_{e \tau} \rightarrow \infty$ ), therefore we may use Equation (1) to study the behavior of the eddy viscosity $\nu_{T}$. If we approximate $d U / d y$ with $u_{\tau} / k y$, and use Eqs. (1) and (23), we obtain

$$
\nu_{T}=\left(k y^{+}-1\right) \nu
$$


This equation shows that the eddy viscosity is $k u_{\tau} y$ for a large $y^{+}$. Now if we model $\nu_{T}$ as

$$
\nu_{T}=C_{\mu} f_{\mu} \frac{K^{2}}{\varepsilon}
$$

and use experimental data to estimate $K \approx 3.5 u_{\tau}^{2}$ in the inertial sublayer, and $\varepsilon \approx u_{\tau}^{3} / k y$, we obtain

$$
\nu_{T}=12.25 C_{\mu} f_{\mu} k y^{+} \nu
$$

Comparing (24) and (26), we obtain

$$
\begin{aligned}
f_{\mu} & =1-\frac{1}{k y^{+}} \\
C_{\mu} & =\frac{1}{12.25} \approx 0.082
\end{aligned}
$$

Eq.(27) shows that the damping function $f_{\mu} \approx 1$ when $y^{+} \geq 30$, which means that the standard $K-\varepsilon$ eddy viscosity model is reasonable in the inertial sublayer, and a significant damping is needed only below $y^{+} \leq 30$, which corresponds to the buffer layer. However, we do not expect that the form of (27) will be appropriate for the buffer layer region, because the assumptions made in these relations are restricted to a large $y^{+}$. Unfortunately, we are not able to find similar analytical expressions like Eqs.(24), (26) in the buffer layer to form the damping function like Eq.(27). We must then depend on the experimental or DNS data to propose appropriate form for $C_{\mu}$ and $f_{\mu}$ to fit Eqs. (23) and (25). Based on the work of Yang and Shih ${ }^{[10]}$, we propose

$$
\begin{aligned}
C_{\mu} & =0.09 \\
f_{\mu} & =\left[1-\exp \left(a_{1} R_{k}+a_{3} R_{k}^{3}+a_{5} R_{k}^{5}\right)\right]^{\frac{1}{2}} \\
a_{1} & =-1.5 * 10^{-4} \quad a_{3}=-1.0 * 10^{-9} \quad a_{5}=-5.0 * 10^{-10} \\
R_{k} & =\frac{K^{1 / 2} y}{\nu}
\end{aligned}
$$

This is no more than a curve fitting based on the solution of DNS of channel flow with $R_{e \tau}=180$. Fortunately, it also works quite well together with the $K-\varepsilon$ equations (described in the next section) for other cases (see section 5). We choose $R_{k}$ in Eq.(28) instead of $y^{+}$ in order to avoid the unphysical behavior of $f_{\mu}$ near the flow separation or reattachment points where the friction velocity $u_{\tau}$ is zero.

\section{4. $K-\varepsilon$ equations}

We may use standard $k-\varepsilon$ model equations for the eddy viscosity model (25), and use Eq.(22) to calculate turbulent mean flows. The boundary conditions can be obtained from Eqs. (14), (16), and (17). The $K-\varepsilon$ equations for incompressible flows can be in general 
modeled as (see Appendix 1 for the derivation of $\varepsilon$ equation):

$$
\begin{aligned}
\frac{D K}{D t}= & {\left[\left(\nu+\frac{\nu_{T}}{\sigma_{k}}\right) K_{, i}\right]_{, i}+\nu_{T} U_{i, j}\left(U_{i, j}+U_{j, i}\right)-\varepsilon } \\
\frac{D \varepsilon}{D t}= & {\left[\left(\nu+\frac{\nu_{T}}{\sigma_{\epsilon}}\right) \varepsilon_{, i}\right]_{, i}+C_{1} f_{1} \nu_{T} U_{i, j}\left(U_{i, j}+U_{j, i}\right) \frac{\varepsilon}{K} } \\
& -C_{2} f_{2} \frac{\varepsilon^{2}}{K}+\nu \nu_{T} U_{i, j k} U_{i, j k}
\end{aligned}
$$

These equations are used only for the flow field outside of the turbulence limit point $y_{\eta}$, where $K_{\eta}$ is non-zero. Therefore, Eq.(30) will not have singularity problems and will not need any near-wall modifications like other $K-\varepsilon$ models do.[11],[12]

As examples, we write the equations and the boundary conditions for both the twodimensional channel and boundary layer flows.

\subsection{Channel flows}

For channel flows, all the quantities are normalized by the friction velocity $u_{\tau}$, and the half width of the channel $h$, and $\nu_{T}$ is normalized by $\nu$.

$$
\begin{aligned}
\frac{\partial U}{\partial t} & =\frac{\partial}{\partial y}\left[\frac{1}{R_{e \tau}}\left(1+\nu_{T}\right) \frac{\partial U}{\partial y}\right]+1 \\
\frac{\partial K}{\partial t} & =\frac{\partial}{\partial y}\left[\frac{1}{R_{e \tau}}\left(1+\frac{\nu_{T}}{\sigma_{k}}\right) \frac{\partial K}{\partial y}\right]+\frac{1}{R_{e \tau}} \nu_{T}\left(\frac{\partial U}{\partial y}\right)^{2}-\varepsilon \\
\frac{\partial \varepsilon}{\partial t} & =\frac{\partial}{\partial y}\left[\frac{1}{R_{e \tau}}\left(1+\frac{\nu_{T}}{\sigma_{\epsilon}}\right) \frac{\partial \varepsilon}{\partial y}\right]+C_{1} f_{1} \frac{1}{R_{e \tau}} \nu_{T}\left(\frac{\partial U}{\partial y}\right)^{2} \frac{\varepsilon}{K}-C_{2} f_{2} \frac{\varepsilon^{2}}{K}+\frac{1}{R_{e \tau}^{2}} \nu_{T} \frac{\partial^{2} U^{2}}{\partial y^{2}}
\end{aligned}
$$

where $R_{e r}$ is a known parameter for channel flows. The boundary conditions for the mean velocity are straightforward:

$$
y=0: \quad U=0, \quad y=1: \quad \frac{\partial U}{\partial y}=0
$$

The boundary conditions for $K$ and $\varepsilon$ must be given at $y_{\eta}$. In the normalized form, $y=y^{+} / R_{e \tau}$. Using Eq.(14), (16) and (17) we obtain

$$
\begin{aligned}
& y=y_{\eta}=\frac{6}{R_{e \tau}}: \quad \varepsilon_{\eta}=0.251 R_{e \tau}, K_{\eta}=0.25 \\
& y=1: \quad \frac{\partial \varepsilon}{\partial y}=0, \frac{\partial K}{\partial y}=0
\end{aligned}
$$

In solving Eq.(31), the eddy viscosity $\nu_{T}$ is negligible when $y \leq y_{\eta}$. 


\subsection{Boundary layer flows}

For a turbulent boundary layer flow, all the quantities are normalized by the free stream velocity $U_{\infty}$, and the length scale $L . \nu_{T}$ is normalized by $\nu$, and the pressure $P$ is normalized by $\rho U_{\infty}^{2}$.

$$
\begin{aligned}
& \frac{D U}{D t}=\frac{\partial}{\partial y}\left[\frac{1}{R_{\infty}}\left(1+\nu_{T}\right) \frac{\partial U}{\partial y}\right]-\frac{\partial P}{\partial x} \\
& \frac{D K}{D t}=\frac{\partial}{\partial y}\left[\frac{1}{R_{\infty}}\left(1+\frac{\nu_{T}}{\sigma_{k}}\right) \frac{\partial K}{\partial y}\right]+\frac{1}{R_{\infty}} \nu_{T}\left(\frac{\partial U}{\partial y}\right)^{2}-\varepsilon \\
& \frac{D \varepsilon}{D t}=\frac{\partial}{\partial y}\left[\frac{1}{R_{\infty}}\left(1+\frac{\nu_{T}}{\sigma_{\epsilon}}\right) \frac{\partial \varepsilon}{\partial y}\right]+C_{1} f_{1} \frac{1}{R_{\infty}} \nu_{T}\left(\frac{\partial U}{\partial y}\right)^{2} \frac{\varepsilon}{K}-C_{2} f_{2} \frac{\varepsilon^{2}}{K}+\frac{1}{R_{\infty}^{2}} \nu_{T} \frac{\partial^{2} U^{2}}{\partial y^{2}}
\end{aligned}
$$

Boundary conditions for the mean velocity are:

$$
y=0: \quad U=0, \quad y=\infty: \quad \frac{\partial U}{\partial y}=0
$$

The boundary conditions for $K$ and $\varepsilon$ are given at $y_{\eta}$. In the normalized form, $y=$ $y^{+} /\left(R_{\infty} u_{\tau}\right)$. Using Eq.(14), (16) and (17) we obtain

$$
\begin{aligned}
& y=y_{\eta}=\frac{6}{R_{\infty} u_{\tau}}: \quad \varepsilon_{\eta}=0.251 R_{\infty} u_{\tau}^{4}, K_{\eta}=0.25 u_{\tau}^{2} \\
& y=\infty: \quad \frac{\partial \varepsilon}{\partial y}=0, \quad \frac{\partial K}{\partial y}=0
\end{aligned}
$$

where the friction velocity is calculated from the solution of the mean velocity.

$$
u_{\tau}=\left[\frac{1}{R_{\infty}} \frac{\partial U}{\partial y}\right]_{y=0}^{\frac{1}{2}}
$$

In solving Eq.(36), the eddy viscosity $\nu_{T}$ is zero when $y \leq y_{\eta}$.

In practical applications, $R_{e \tau}$ and $R_{e \infty}$ are large numbers, hence $y_{\eta}$ is usually very small. Therefore, as an approximation we may let $y_{\eta}=0$, but $\varepsilon_{\eta}$ and $K_{\eta}$ must be given by Eqs.(35) and (40) respectively. These equations have been applied to the calculations shown in the next section.

\section{Comparison of models}

To compare the present model with the DNS data and other models (e.g. Jones and Launder ${ }^{[11]}$, and Chien $\left.{ }^{[12]}\right)$, we have made calculations on two channel flows $s^{[0],[7]}$ and two boundary layer flows ${ }^{[8],[15]}$. In the present model, all the model constants are the same as used in the standard $K-\varepsilon$ model ${ }^{[13]}$. Therefore the present model will also be suitable for flows far from the wall. The other two models used here for comparison do not have 
this property. Results are shown in figures $2-5$. In figure 2 and figure 3 , three models are compared with two DNS data for channel flows: one with $R_{e \tau}=180$, the other with $R_{e \tau}=395$. The profiles of mean velocity, Reynolds stress, turbulent kinetic energy and its dissipation rate are plotted in these figures. The present model is significantly better than the other two models. Figure 4 shows the similar comparison for a turbulent boundary with $R_{e \theta}=1410$. The agreement between the present model and DNS data is excellent. Figure 5 shows the results compared with Klebanoff ${ }^{[15]}$ and other boundary layer experiments. The skin friction from DNS data ${ }^{[8]}$ is also shown in this figure. The results of present model are more consistent with the DNS data than the experiments.

It is also worthwhile to emphasize that the present model equations with the standard model coefficients have the simplest form among all two-equation models. Hence, we expect that they will have less numerical stiffness in complex turbulent flows.

\section{Appendix 1. $K-\varepsilon$ equations}

The equation for the dissipation rate of turbulent kinetic energy $\varepsilon$ is proposed following Lumley[14]:

$$
\frac{D \varepsilon}{D t}=\left[\left(\nu+\frac{\nu_{T}}{\sigma_{\varepsilon}}\right) \varepsilon_{, i}\right]_{, i}-\frac{\varepsilon^{2}}{K} \Psi
$$

where $\Psi$ stands for the entire mechanism of the production and destruction of the dissipation rate $\varepsilon$. At the level of the $K-\varepsilon$ model, we assume that $\Psi$ is a function of $\nu, \nu_{T}, K, \varepsilon, U_{i, j}$, and $U_{i, j k}$. Because $\Psi$ is an invariant, it must be a function of the invariants that can be constructed from these quantities: $R_{t}, \nu_{T} U_{i, j} U_{i, j} / \varepsilon$, and $\nu \nu_{T} U_{i, j k} U_{i, j k} / \varepsilon^{2}$, where $R_{t}$ is the turbulent Reynolds number $\frac{K^{2}}{\nu \varepsilon}$. We now expand $\Psi$ in a Taylor series about these invariants and keep only the linear terms to obtain

$$
\Psi=\psi_{0}+\psi_{1} \frac{\nu_{T} U_{i, j} U_{i, j}}{\varepsilon}+\psi_{2} \nu_{T} U_{i, j k} U_{i, j k} \frac{K}{\varepsilon^{2}}
$$

where the coefficients $\psi_{0}, \psi_{1}$ and $\psi_{2}$ are in general function of $R_{t}$. Inserting (A2) into (A1), we obtain

$$
\begin{aligned}
\frac{D \varepsilon}{D t}= & {\left[\left(\nu+\frac{\nu_{T}}{\sigma_{\epsilon}}\right) \varepsilon_{, i}\right]_{, i}+C_{1} f_{1} \nu_{T} U_{i, j}\left(U_{i, j}+U_{j, i}\right) \frac{\varepsilon}{K} } \\
& -C_{2} f_{2} \frac{\varepsilon^{2}}{K}+\nu \nu_{T} U_{i, j k} U_{i, j k}
\end{aligned}
$$

where $C_{1}, C_{2}$ are model constants, and $f_{1}, f_{2}$ are in general functions of $R_{t}$, and $\psi_{2}=-1$. The forms of $C_{1}, C_{2}$ and $f_{1}, f_{2}$ are chosen to be the same forms as used in the standard $K-\varepsilon$ model:

$$
\begin{aligned}
C_{1} & =1.44, \quad C_{2}=1.92 \\
f_{1} & =1, \quad f_{2}=1-0.22 \exp \left(-\frac{R_{t}^{2}}{36}\right) \\
\sigma_{k} & =1, \quad \sigma_{\epsilon}=1.3
\end{aligned}
$$




\section{References}

[1] Hanjalic, K. and Launder, B.E., "Contribution Towards a Reynolds-stress Closure for Low-Reynolds-Number Turbulence," J. Fluid Mech. 74. April 1976, pp. 593-619.

[2] Patel, V.C., Rodi, W. and Scheuerer, G., "Turbulence models for near-wall and lowReynolds-number flows: A review," AIAA Journal, 23, 1985, pp. 1308-1319.

[3] Mansour, N.N., Kim,J. and Moin. P., "Reynolds-Stress and Dissipation Rate Budgets in a Turbulent Channel Flow," J. Fluid Mech., Vol. 194, 1988, pp. 15-44.

[4] Lai, Y.G. and So, R.M.C., "On near-wall turbulent flow modeling," J. Fluid Mech. (1990), vol.221, pp.641-673.

[5] Shih, T. -H., "An Improved $k-\epsilon$ Model for Near-Wall Turbulence and Comparison with Direct Numerical Simulation," NASA TM-103221, August 1990.

[6] Kim, J., Moin, P. and Moser, R., "Turbulent Statistics in fully Developed Channel Flow at Low Reynolds Number," Journal of Fluid Mechanics, 177, pp. 133-166, 1987.

[7] Kim, J., Personal communication, 1992.

[8] Spalart, P. R., "Direct Simulation of a Turbulent Boundary Layer up to $R e_{\theta}=1410$," Journal of Fluid Mechanics, Vol. 187, pp. 61-98, 1988.

[9] Tennekes, H. and Lumley, J.L., A First Course in Turbulence, The MIT Press.

[10] Yang, Z. and Shih, T. -H., "A $k-\epsilon$ Modeling of Near Wall Turbulence,"Proceedings of 4th International Symposium on Computational Fluid Dynamics, UC Davis, 1991.

[11] Jones, W. P. and Launder, B. E., "The Calculation of Low-Reynolds Number Phenomena with a Two-Equation Model of Turbulence, "International Journal of Heat and Mass Transfer, Vol. 16, pp. 1119-1130, 1973.

[12] Chien, K.-Y., "Predictions of Channel and Boundary-Layer Flows with a Low Reynolds Number Turbulence Model," AIAA Journal, Vol. 20, pp. 33-38, Jan 1982.

[13] Launder, B.E. and Spalding, D.B., "Computer Methods in Applied Mechanics and Engineering, 3, 269 (1974).

[14] Lumley, J.L., "Computational modeling of turbulent flows," Adv. AppI. Mech. 1978, $18,123$.

[15] Klebanoff, P. S., "Characteristics of Turbulence in a Boundary Layer with Zero Pressure Gradient," NACA-TN-3178, 1954. 


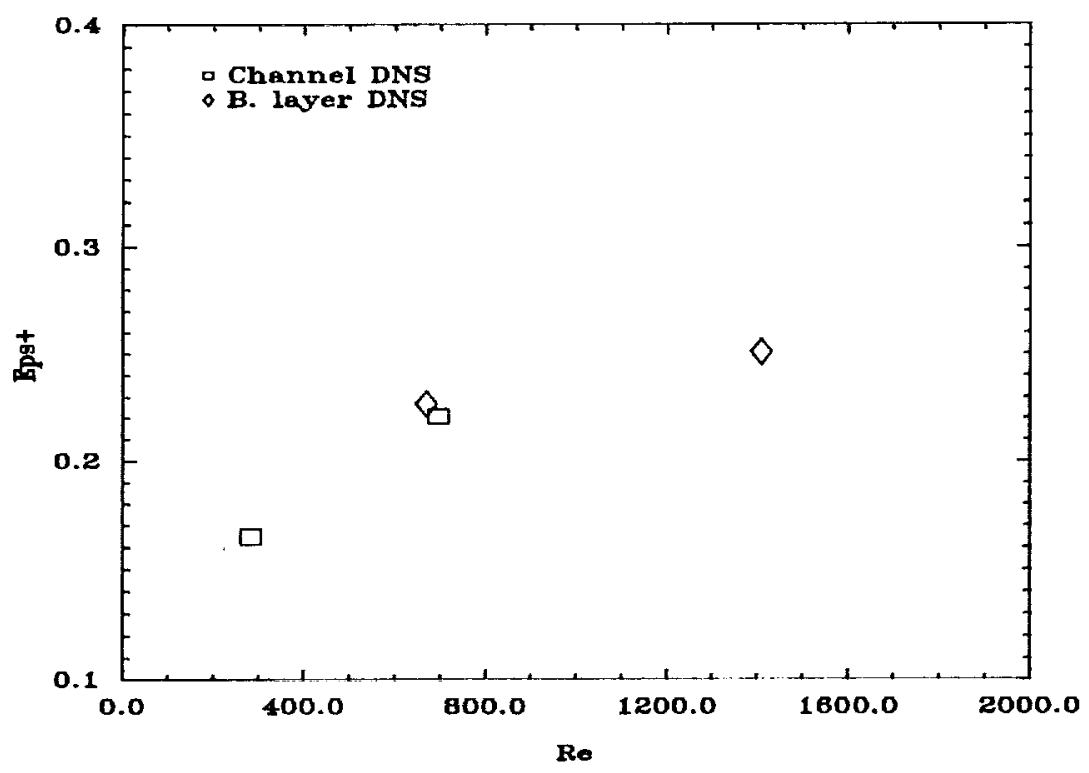

Figure 1. The asymptotic value of the dissipation rate at high $R_{e \theta}$. 

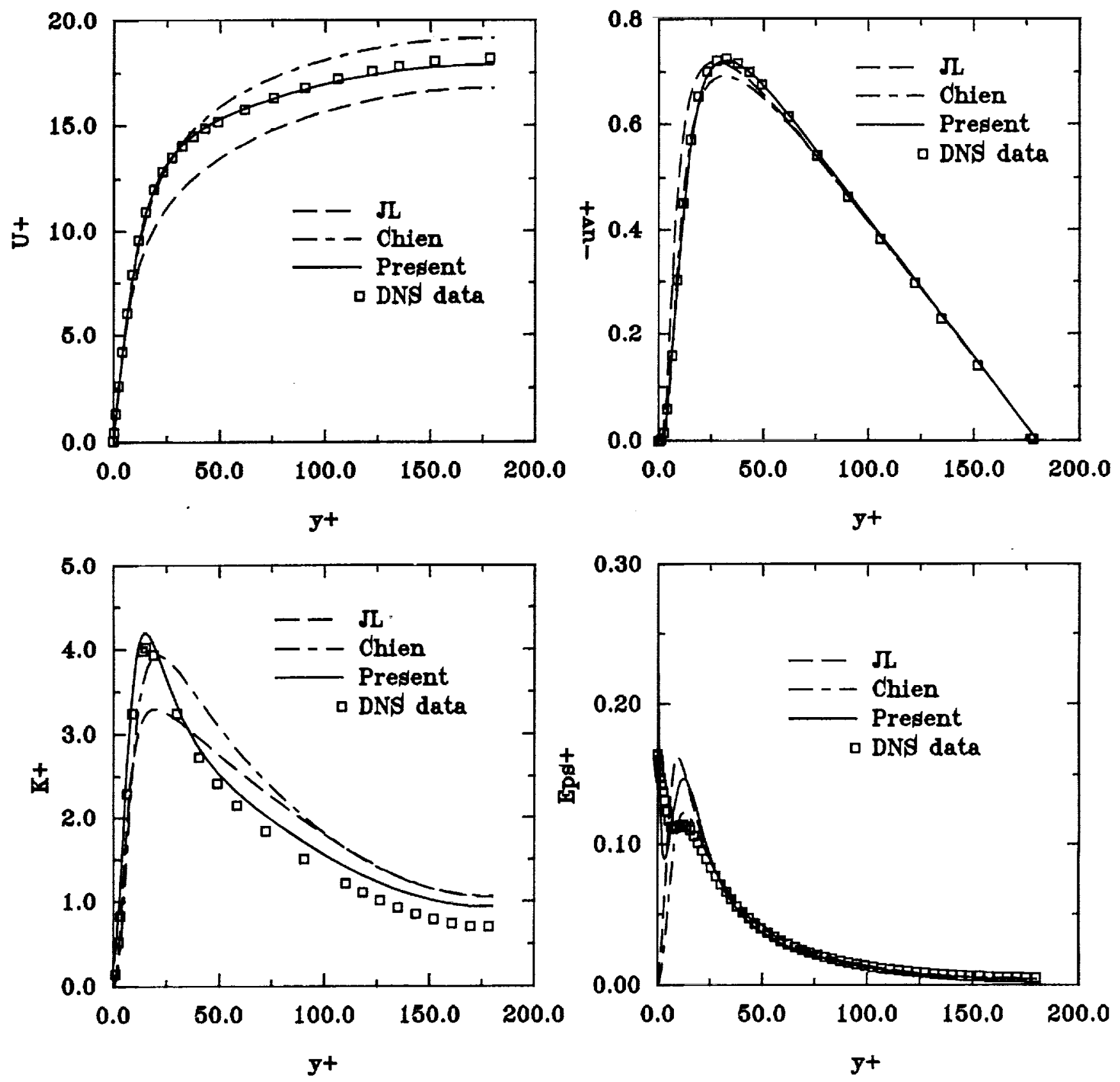

Figure 2. Comparison of models with DNS of 2-D channel with $R_{e \tau}=180$. 

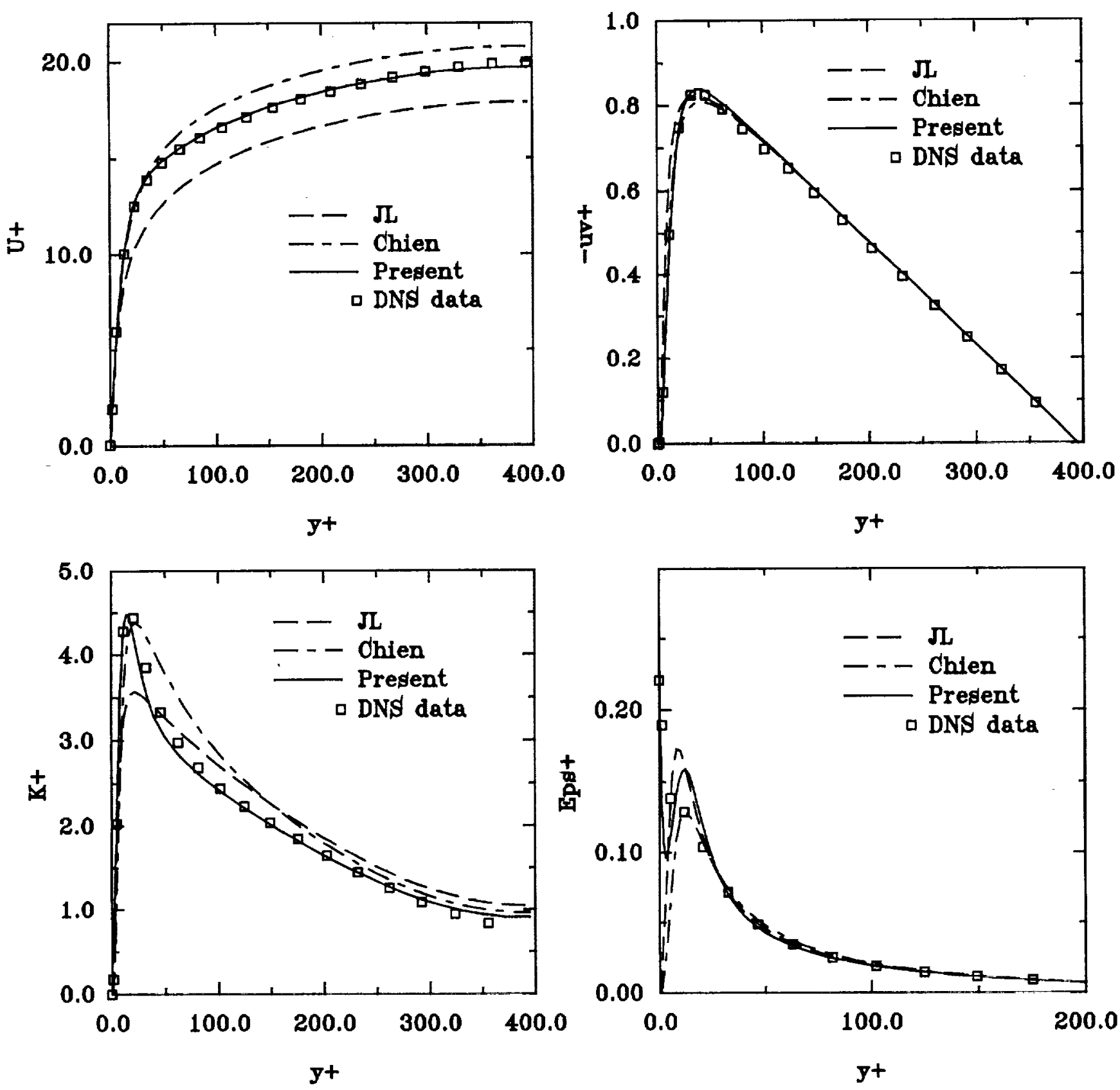

Figure 3. Comparison of models with DNS of 2-D channel with $R_{e \tau}=395$. 

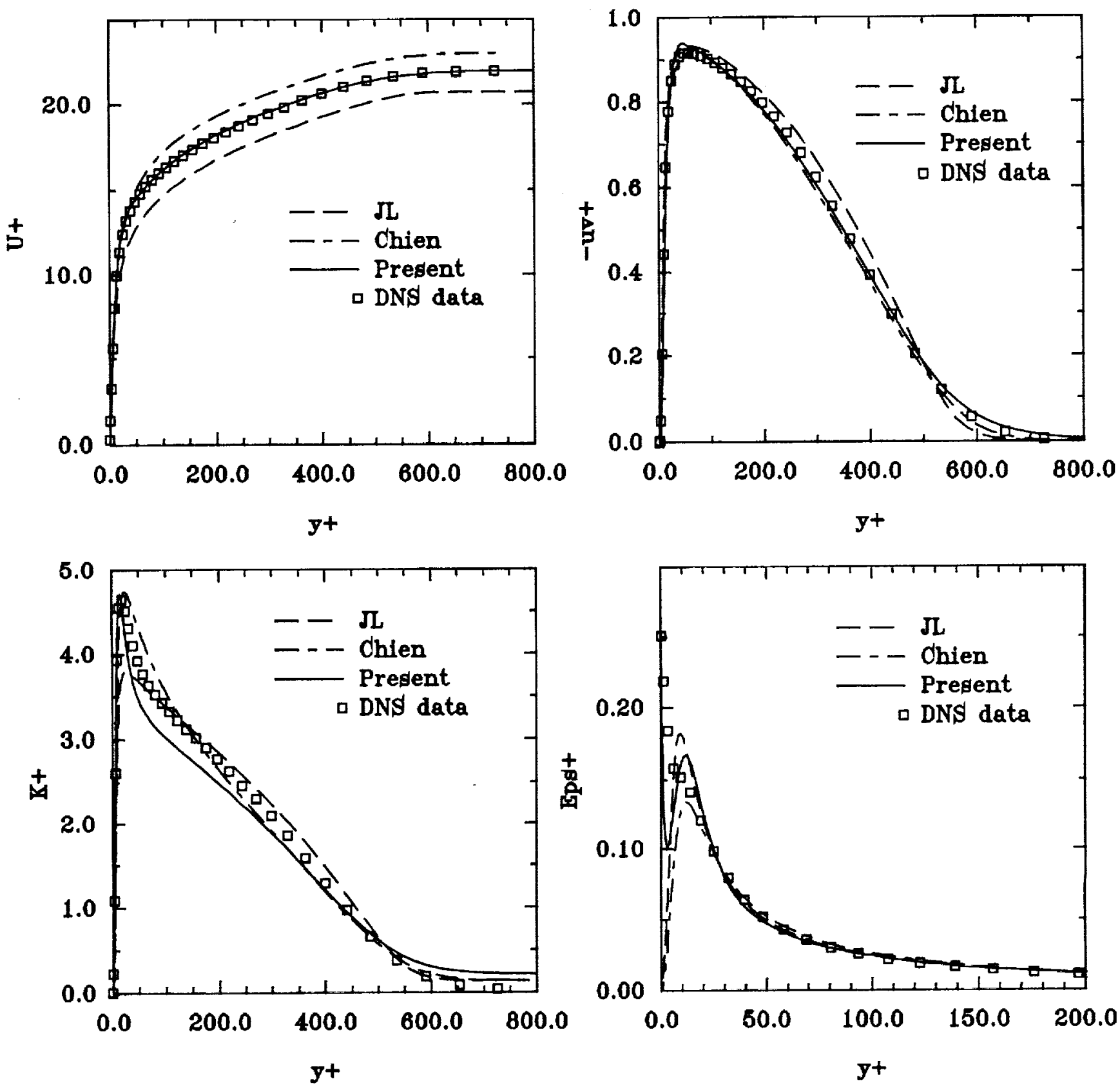

Figure 4. Comparison of models with DNS of 2-D boundary layer flow with $R_{e \theta}=1410$. 

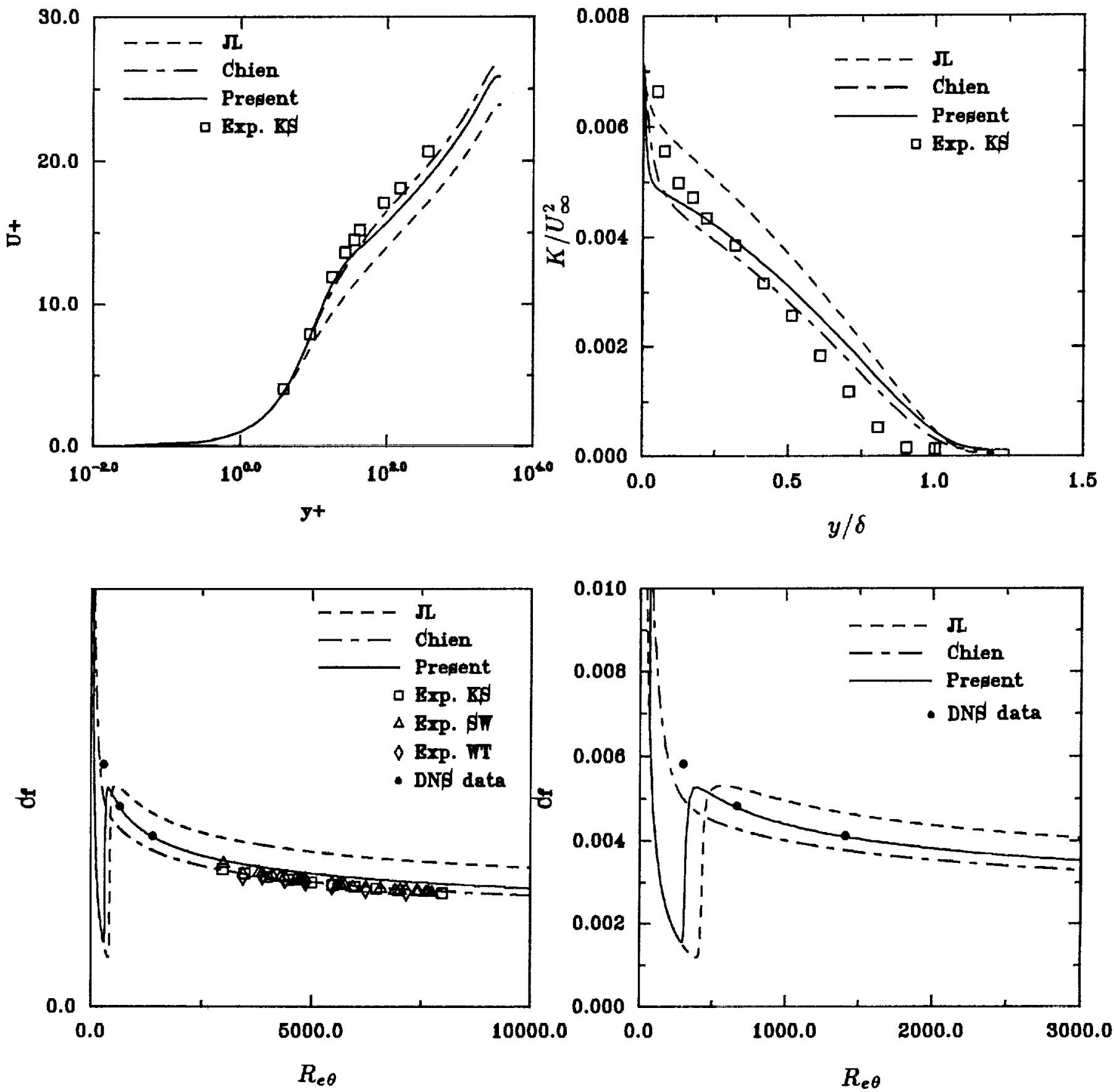

Figure 5. Comparison of models with the experiments of 2-D boundary layer flows (Klebanoff ${ }^{13]}$ and others). 


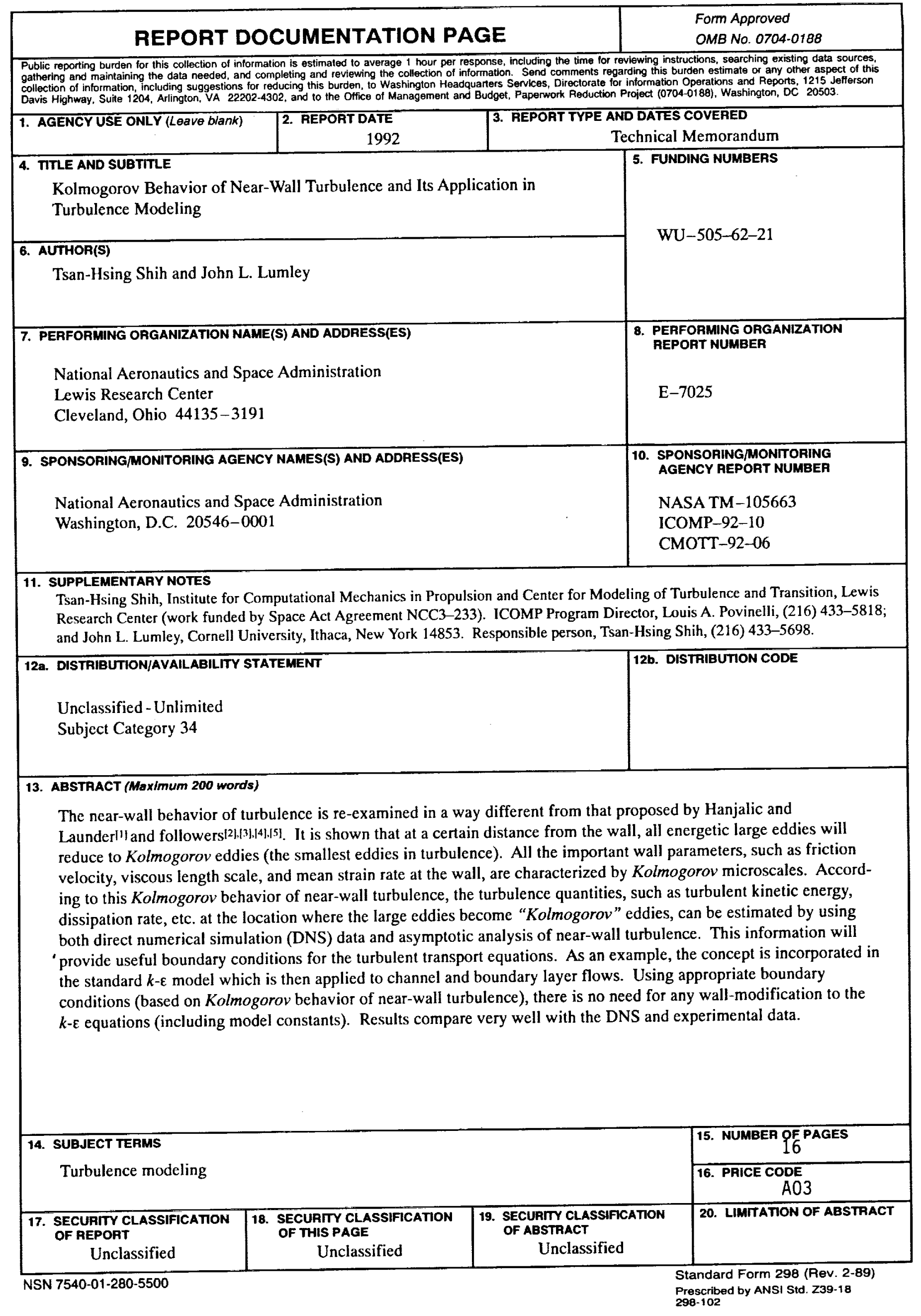

